\title{
The RNA interference revolution
}

G. Lenz

\section{Correspondence}

G. Lenz

Departamento de Biofísica, UFRGS

Av. Bento Gonçalves, 9500

91501-370 Porto Alegre, RS

Brasil

Fax: +55-51-3316-7003

E-mail: lenz@ufrgs.br

Received February 16, 2005

Accepted August 11, 2005
Departamento de Biofísica, Universidade Federal do Rio Grande do Sul, Porto Alegre, RS, Brasil

\begin{abstract}
The discovery of double-stranded RNA-mediated gene silencing has rapidly led to its use as a method of choice for blocking a gene, and has turned it into one of the most discussed topics in cell biology. Although still in its infancy, the field of RNA interference has already produced a vast array of results, mainly in Caenorhabditis elegans, but recently also in mammalian systems. Micro-RNAs are short hairpins of RNA capable of blocking translation, which are transcribed from genomic DNA and are implicated in several aspects from development to cell signaling. The present review discusses the main methods used for gene silencing in cell culture and animal models, including the selection of target sequences, delivery methods and strategies for a successful silencing. Expected developments are briefly discussed, ranging from reverse genetics to therapeutics. Thus, the development of the new paradigm of RNA-mediated gene silencing has produced two important advances: knowledge of a basic cellular mechanism present in the majority of eukaryotic cells and access to a potent and specific new method for gene silencing.
\end{abstract}

Key words

- RNAi

- siRNA

- miRNA

- Gene silencing

- Knockdown

\section{Basic mechanism of RNA-mediated gene silencing}

RNA silencing has long been recognized as part of the immune response against viruses in plants (1), but the RNA interference (RNAi) revolution started with the observations that sense plus antisense RNA were much more effective in silencing a gene in Caenorhabditis elegans when compared to the traditionally used antisense strand (2). This finding, together with the discovery that the genes of $C$. elegans feeding on bacteria expressing double-stranded RNA (dsRNA) were silenced in the whole organism (3), were pivotal for the explosion that we see today in the studies using RNAi $(4,5)$. In 2001, nearly half of all papers on RNAi had C. elegans as a model, whereas today this number is about $10 \%$, indicating the fast transfer of the paradigm from the nematode community to the rest of the biological sciences.

When dsRNAs of various lengths are produced by the cell or introduced into the cell, they are processed by the dsRNA endoribonuclease Dicer into 21-nt smallinterfering RNAs (siRNAs; Table 1). These siRNAs in turn associate with an RNAiinducing silencing complex (RISC) and direct the destruction of mRNA complementary to one of the strands of the siRNA. RISC cleaves the target mRNA in the middle of the complementary region, ten nucleotides upstream of the nucleotide paired with the $5^{\prime}$ end of the guide siRNA. 
Annealing of one strand of the siRNA to the target mRNA molecule allows an RNAdirected RNA polymerase (RdRP) to use the target mRNA as a template and the siRNA as a primer in order to produce a new doublestranded mRNA, which can be cleaved by Dicer, thus contributing to the pool of siRNAs (6). This positive feedback seems to be fundamental for the high efficiency of the RNAi process.

With the discovery of RNAi, it was rapidly realized that endogenous "genes" can also produce double-stranded hairpins that interfere with the function of specific genes. These endogenous siRNAs, which were called micro-RNAs (miRNAs), affect mainly translational efficiency rather than mRNA stability. Studies on mammalian systems confirmed the $C$. elegans data, indicating that this class of RNAs is involved in the major biological events from development to control of metabolism (7). In an example of the surprises that lay ahead, an evolutionarily conserved miRNA expressed specifically in pancreatic islets did suppress glucose-induced insulin secretion, potentially playing a role in diabetes (8). Additionally, viruses seem to employ miRNAs as part of their strategy to infect host cells and replicate, since several miRNAs were identified in the genome of the Epstein-Barr virus (9). This opens a completely new window into the study of the mechanism of viral infections and allows the development of new treatment strategies.

RNAi does not work well in all cell types. Neurons from C. elegans, for example, are only slightly affected by systemic RNAi. This seems to be due, at least in part, to the expression of a dsRNA-specific RNAse, called ERI-1, and its ablation increased the interference process in neurons of C. elegans (10). A deeper understanding of the mechanisms negatively regulating RNAi may contribute to ways of artificially increasing the efficiency of the RNAi process.

In an indication that the basic rules are far from understood, Kawasaki and Taira (11) showed that siRNAs can reduce the transcription of the target gene by directing DNA methylation. With this discovery, dsRNAs were shown to be involved in the main processes that control protein production: RNA synthesis, RNA degradation and protein synthesis.

\section{Successful gene silencing}

\section{Selection of the target sequence}

In mammals, the introduction of dsRNAs longer than $30 \mathrm{bp}$ activates antiviral pathways, leading to nonspecific inhibition of translation and cytotoxic responses (12).

\begin{tabular}{|c|c|c|}
\hline Abbreviation & Meaning & Function \\
\hline Dicer & & $\begin{array}{l}\text { Cytoplasmic RNAse-III-like enzyme that transforms } \\
\text { dsRNAs into siRNAS of } \sim 21 \mathrm{nt}\end{array}$ \\
\hline DsRNA & Double-stranded RNA & Induces RNAi \\
\hline MiRNA & micro-RNA & $\begin{array}{l}\text { siRNA found in the genome that blocks translation } \\
\text { elongation or termination }\end{array}$ \\
\hline RISC & RNA-induced silencing complex & $\begin{array}{l}\text { Single stranded-RNA containing the siRNA protein } \\
\text { complex that mediates mRNA degradation }\end{array}$ \\
\hline RNAi & RNA interference & The silencing process \\
\hline ShRNA & Short-hairpin RNA & $\begin{array}{l}\text { siRNA produced by the transcription of RNA } \\
\text { polymerase III from a U6- or } \mathrm{H} 1 \text {-driven promoter in } \\
\text { a plasmid }\end{array}$ \\
\hline SiRNA & Small-interfering RNA & dsRNAs that mediate mRNA degradation \\
\hline
\end{tabular}


Therefore, the use of long dsRNAs was abandoned. Thus, today, RNAi is elicited almost exclusively with 21-nt dsRNAs, requiring the selection of a short sequence of the mRNA to be targeted. Despite several advances in the understanding of the silencing mechanism, there is no guarantee that a given sequence of $21 \mathrm{nt}$ will work, but several rules, mainly based on trial and error rather than on mechanistic understanding, help to increase the likelihood of success.

Table 2 contains a list of rules that help decide which 21-nt sequence is more likely to produce an efficient silencing. Usually, it is interesting to start with the search for a sequence of $19 \mathrm{nt}$ that is flanked by AA on the $5^{\prime}$ end and TT of the 3 ' end of the sense strand (rule 1), and with a GC content of 40 to $60 \%$ (rule 2). Targeting of the first and last $100 \mathrm{nt}$ of the mRNA traditionally has not produced a good silencing (rule 3 ).

The molecular mechanism which probably contributes more to the efficiency of the silencing process is an amplification catalyzed by an RdRP (6). Since RNA polymerization occurs always by extending the primer in the 5' to 3' direction, the more towards the $3^{\prime}$ end of the mRNA the siRNA primer anneals, the longer is the dsRNA produced by the RdRP and therefore, theoretically, the more efficient the silencing process (rule 4). There does not seem to be any difference in efficiency between targeting the transcribed or the untranscribed region (UTR) of the mRNA, and therefore the 3'UTR is a good choice for targeting, even more so if rescue experiments using the gene ectopically expressed in a plasmid are used. In this case, the plasmid would not have the 3'UTR and, if the rescue works, this would indicate that the effect is due to the down-regulation of the target gene (rule 5). The other rescue experiment that can be conducted is to transfect a plasmid with silent mutations at the sequence targeted by RNAi, right in the middle of the sequence. This should not be affected by the RNAi and therefore should rescue the phenotype. Careful rescue experiments have indicated that a 21-bp dsRNA does indeed induce cytotoxicity in cell culture and that a 19-bp dsRNA does not, but still retains the silencing ability (13).

Which of the two strands of the siRNA is loaded into the RISC complex is pivotal for the success of the interference, since only the antisense one can pair with the mRNA and induce its degradation. The higher the CG content of the $5^{\prime}$ end when compared to the $3^{\prime}$ end of the sense strand, the higher the likelihood of success of the antisense to be used (14). Therefore, the ideal sequences contain 4 CGs at the $5^{\prime}$ end and 4 AUs at the 3 ' end of the sense strand (rule 6). Interestingly, this strand bias was also observed in endogenous miRNAs (15).

RNAi was first regarded as an absolutely specific way of down-regulating a gene, but gene array studies indicated that the specificity depends on the position and the number of mismatches. The selected sequence should not be identical in the central core of 14-15 nt to any off-target sequence, although, again, there is no certainty if a given sequence will only affect the target sequence (16). Therefore, a BLAST search against the genome of the species in which the siRNA will be used is fundamental for a good specificity (rule 7). The use of more than one possible siRNA sequence is becoming a

Table 2. Some rules for a successful sequence selection.

$\begin{array}{ll}\text { Rule 1 } & \text { AA (N19) TT } \\ \text { Rule 2 } & \text { GC content from } 40 \text { to } 60 \% \\ \text { Rule 3 } & \text { Not in the first and last } 100 \text { bp of RNA } \\ \text { Rule } 4 & \text { Towards the 3' end of mRNA } \\ \text { Rule 5 } & \text { Plan rescue experiments } \\ \text { Rule 6 } & \text { GC content on 5' end higher than on 3' end } \\ \text { Rule 7 } & \text { BLAST for specificity } \\ \text { Rule } 8 & \text { Avoid stretches of a single nucleotide, especially Ts, when using } \\ & \text { short-hairpin RNAs } \\ \text { Rule 9 } & \text { Check your selection against the selection of websites that predict } \\ & \text { targets: } \\ & \text { http://web.mit.edu/mmcmanus/www/home1.2files/siRNAs.htm } \\ & \text { http://hydra1.wistar.upenn.edu/Projects/siRNA/siRNAindex.htm } \\ & \text { http://www.cshl.edu/public/SCIENCE/hannon.html }\end{array}$


standard requirement of experiments involving RNAi. Additionally, a control with a scrambled sequence, or a sequence directed at a gene not present in the genome of the organism studied, such as green fluorescent protein (GFP), is also important.

Still, RNAi is among the most specific methods available for the ablation of genes, and, when appropriately used, a single nucleotide mismatch can be sufficient for discrimination between the target and the off-target sequence, as exemplified with the oncogene RasV12 and its proto-oncogene Ras (17).

Despite the use of these rules, there is no guarantee that a given sequence will work, and only careful testing and the use of more than one sequence can make the silencing process to be more successful and accurate.

\section{Delivery}

Bacteria in C. elegans. The discovery that probably contributed most to the rapid success of RNAi technology was the effective silencing obtained by feeding $C$. elegans with bacteria containing a plasmid which produces siRNA (3). Surprisingly, siRNA spreads to all cells of the nematode, except some neuronal cells, although strains were developed in which the RNAi is more effective, also working in neurons (10).

Double-stranded RNAs. siRNA delivery is more complicated in mammals than in nematodes and evidence that RNAi would also work in mammals started to appear in 2001, with the use of 21- and 22-nt siRNAs, transfected directly into human cell lines. These siRNAs were able to reduce very efficiently and specifically the expression of several genes in various cell lines (18). Although very efficient, direct transfection of the siRNA is more costly when compared with other methods of delivery. Stability has being improved considerably, and recently direct siRNA delivery into the circulation of mice proved to be quite efficient in reducing cholesterol. These siRNAs were synthesized with a phosphorothioated backbone and 2'$\mathrm{O}$-methyl ribose on the sense and anti-sense strands in order to enhance resistance to degradation by exo- and endonucleases (19).

Plasmids. In few instances in the history of science were so many discoveries made simultaneously as in the RNAi field. This applies mainly to methodological advances, as was the case for plasmid delivery of shorthairpin RNA (shRNA; see Table 3, with at least five papers published in April and May 2002 showing the production of RNAi by shRNA generated by a plasmid.

Two promoters of the RNA polymerase III, called U6 and H1, have been widely used to produce shRNA. These promoters differ in size, but contain the same conserved sequences and apparently there is no significant difference in the efficiency of silencing induced by these promoters (20). The stop codon for RNA polymerase III is a sequence of five Ts (21), and therefore, if using shRNAs, stretches of more than 4 Ts should be avoided (rule 8; Figure 1).

Viruses and knockdowns. The description of shRNA produced by plasmids was followed rapidly by many papers describing shRNA-producing viral vectors (see Table 3). Several of these papers also described stable insertion of the RNAi into the genome and maintenance during development, thus creating animals with constitutively expressed siRNA. These animals showed a reduction of the target gene in the $90 \%$ range, depending on the tissue and the siRNA used and therefore were termed knockdowns, to differentiate them from knockouts.

The lack of total suppression of the expression of a given RNA actually can be used to an advantage. An siRNA that presented a very strong down-regulation, produced animals with a phenotype identical to that of knockout animals, whereas a less efficient siRNA produced a phenotype similar to that of $+/-$ animals (22). The differential efficiency of siRNAs can be used to 
produce a whole range of down-regulation, being useful to study the effects of small changes in the expression of a given gene, which cannot be determined with traditional genetics.

Another welcome development is the establishment of shRNA-producing vectors that can be regulated. These vectors were adapted from the Cre-Lox system, in which the whole shRNA expressing cassette was loxed, and therefore the production of RNAi can be inactivated by the presence of Cre (23). On the other hand, the insertion of a loxed GFP gene between the U6 promoter and the siRNA insertion created a construct that only produces shRNAs in the presence of Cre. Ani- mals containing these genes can be easily crossed to animals expressing the recombinase Cre under the regulation of cell-specific promoters, thus producing animals with knockdown in specific cells, or, a more difficult result to obtain with standard knockout techniques, having knockdown in all cells except in a specific subset. Additionally, a system that allows an even tighter regulation was developed by Wiznerowicz and Trono (24), who used the tetracyclineregulated system to produce siRNAs only in the presence of doxycycline.

Comparison of delivery methods. The choice of delivery methods depends on the system used and the questions asked. When

Table 3. A short history of RNAi.

\begin{tabular}{|c|c|c|}
\hline Discovery & $M / Y$ & Authors \\
\hline Double-stranded RNA (dsRNA) works better than anti-sense RNA & 2/1998 & Fire et al. (2) \\
\hline Feeding C. elegans with bacteria expressing dsRNAs & $10 / 1998$ & Timmons and Fire (3) \\
\hline Micro-RNAs & $\begin{array}{r}2 / 2000 \\
10 / 2001 \\
10 / 2001\end{array}$ & $\begin{array}{l}\text { Reinhart et al. (7) } \\
\text { Lau et al. (41) } \\
\text { Lee and Ambros (42) }\end{array}$ \\
\hline RNA interference (RNAi) in mammalian cell culture & $5 / 2001$ & Elbashir et al. (18) \\
\hline RNAi delivered by plasmids (short-hairpin RNA, shRNA) & $\begin{array}{l}2 / 2002 \\
4 / 2002 \\
4 / 2002 \\
5 / 2002 \\
5 / 2002 \\
5 / 2002\end{array}$ & $\begin{array}{l}\text { Paddison et al. (43) } \\
\text { Blummelkamp et al. (44) } \\
\text { Paddison et al. (45) } \\
\text { Miyagishi and Taira (46) } \\
\text { Lee et al. (47) } \\
\text { Paul et al. (48) }\end{array}$ \\
\hline Viral delivered shRNA & $\begin{array}{r}9 / 2002 \\
10 / 2002\end{array}$ & $\begin{array}{l}\text { Blummelkamp et al. (17) } \\
\text { Xia et al. (49) }\end{array}$ \\
\hline Viral shRNA-induced animal knockdowns & $\begin{array}{l}3 / 2003 \\
4 / 2003\end{array}$ & $\begin{array}{l}\text { Rubinson et al. (25) } \\
\text { Stewart et al. (50) }\end{array}$ \\
\hline C. elegans genome screen using RNAi & $\begin{array}{l}1 / 2003 \\
1 / 2003\end{array}$ & $\begin{array}{l}\text { Kamath et al. (26) } \\
\text { Ashrafi et al. (27) }\end{array}$ \\
\hline Human genome screen using RNAi & $\begin{array}{r}10 / 2003 \\
3 / 2004\end{array}$ & $\begin{array}{l}\text { Kiger et al. (28) } \\
\text { Berns et al. (29) }\end{array}$ \\
\hline Enzyme-mediated RNAi library synthesis & $\begin{array}{l}2 / 2004 \\
2 / 2004 \\
4 / 2004\end{array}$ & $\begin{array}{l}\text { Sen et al. (30) } \\
\text { Shirane et al. (31) } \\
\text { Luo et al. (32) }\end{array}$ \\
\hline
\end{tabular}

aln this report, a large piece of dsRNA produced by a plasmid was used; therefore, this does not technically qualify as an shRNA, but certainly contributed to the idea of producing shRNA, as indicated by the subsequent publication by the same group . 


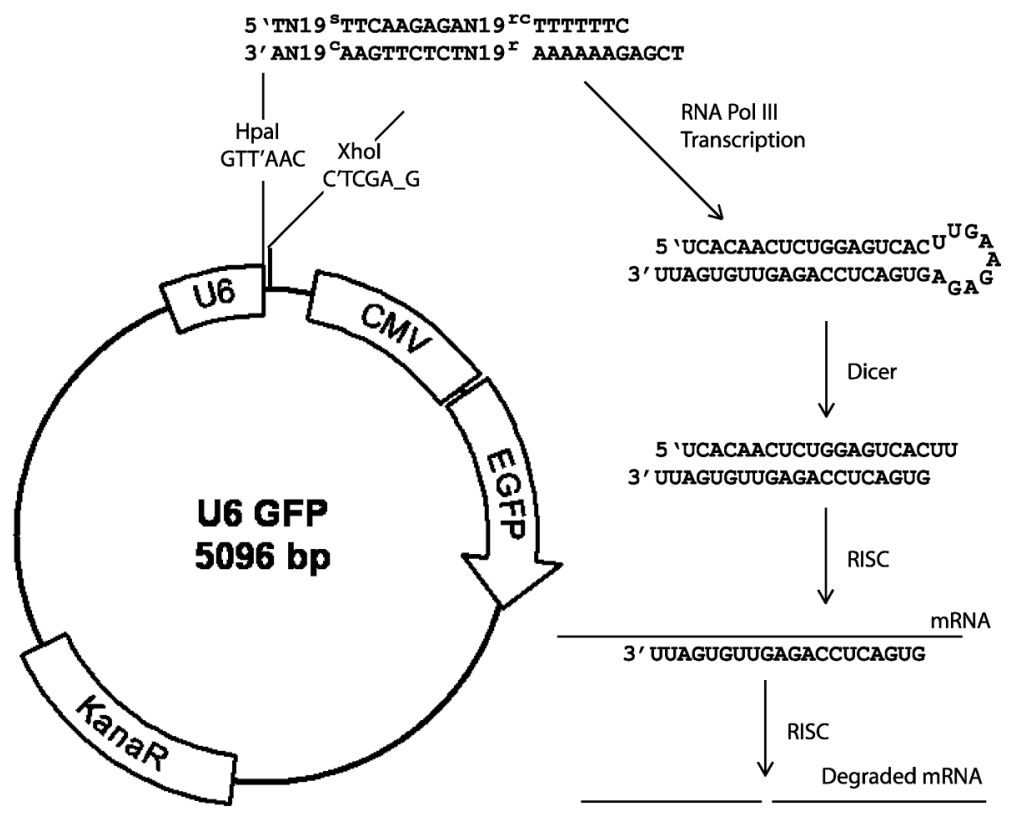

Figure 1. Plasmid-mediated RNA interference. Basic components of the small-interfering RNA-producing plasmid include an RNA polymerase III (RNA Pol III) promoter (U6 or H1) upstream of the insert site of the dsDNA, an indication (GFP, green fluorescent protein) and a selection marker (KanaR/ NeoR). CMV - cytomegalovirus; EGFP = enhanced green fluorescent protein; RISC = RNA-induced silicencing complex; $s=$ sense; $r=$ reverse; $c=$ complement; $r c=$ reverse complement. Map generated in pDraw32 (www.acaclone.com).

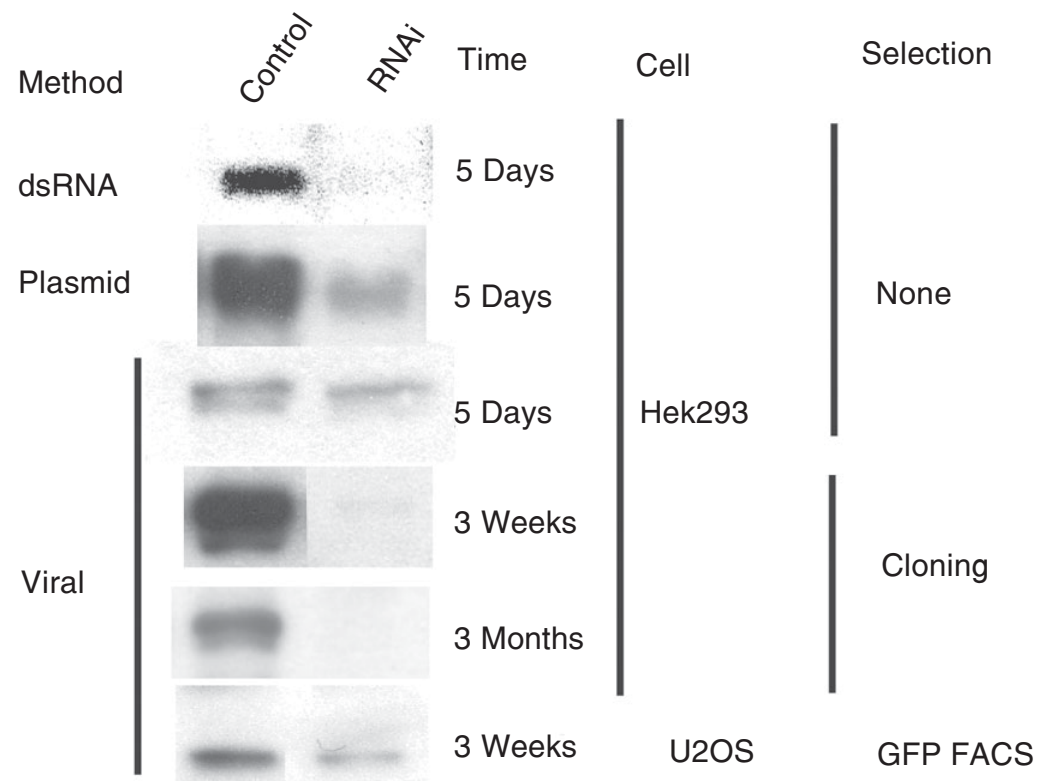

Figure 2. Small-interfering RNAs targeted against a kinase involved in mitosis were introduced into a mammalian cell culture either by direct transfection of plasmids or by means of viral transduction. An analysis of time, cell type and selection method is discussed in the text. dsRNA = double-stranded RNA; RNAi = RNA interference; GFP = green fluorescent protein; FACS = flow cytometry. a fast and efficient method is required, direct transfection of 21-nt dsRNA probably is the method of choice. Its transfection efficiency is much higher than plasmid transfection, targeting almost $100 \%$ of the majority of cell types in culture. An indication that shRNA works as efficiently as dsRNA is the observation that viral mediated transduction and selection of the positive cells by cloning was able to reduce the amount of the protein to an almost undetectable level, similarly to dsRNA transfection. The advantage of using lentiviral vectors is that the silencing is stable (25). Several results indicate that RNAi is affected by differences in cell type. This is based on the observations that $C$. elegans silencing occurs in all cells except neurons and that transgenic mice expressing siRNAproducing constructs show differential reduction of the target gene depending on the cell type (25). This is also seen in cell culture, as shown in Figure 2. Although the selection methods for Hek293 and for U2OS were different, the latter were selected to $99.9 \%$ purity, and the reduction in the target gene was still not higher than $60 \%$.

Plasmid transfection and viral transduction seem to work similarly, but lentiviral transduction has the advantage of stably integrating into the genome and therefore making the selection and production of stable cell lines more efficient.

\section{Applications}

\section{Reverse genetics or whole genome screens}

The ease of using RNAi in nematodes has greatly simplified reverse genetics and is now a standard in $C$. elegans genetics. This technique was used to ablate all genes in the C. elegans genome (26) and bacteria expressing siRNA against all genes are now commercially available. Ashrafi et al. (27) showed that this technique was very efficient for the study of genes involved in fat metabolism, confirming several genes al- 
ready known to be involved in this process, but also finding several others not previously linked to the control of fat metabolism.

Two screens designed to silence a large number of genes of the human genome were performed in cell culture. Kiger et al. (28) used direct transfection of dsRNA targeted against approximately 1000 genes and analyzed the effect of knockdown of these gene on the shape of cells in culture. Berns et al. (29) employed a set of more than 23 thousand distinct shRNAs using retroviral vectors, targeting almost 8 thousand different human genes in order to find new components of the p53 signaling pathway.

These screenings were done by individually producing the siRNAs, at quite considerable cost. At the beginning of 2004, three papers were published describing an enzymatic process for the production of an siRNA library (Table 2). Interestingly, all three papers used an almost identical process for the library synthesis, including the same restriction enzyme, MmeI, which has the exquisite capacity to cleave 20-21 bp away from its recognition site (30-32). Improvements of these techniques will certainly produce the methodology needed for an easy construction of siRNA-producing libraries and consequently permit many screenings in mammalian systems.

\section{Therapeutics}

The results obtained with animal models are very encouraging. Direct injection into the tail vein of mice of siRNA targeted against apolipoprotein $\mathrm{B}$ reduced the cholesterol levels by 60 to $80 \%$. In addition to chemically increasing stability, as mentioned before, the sense strand was conjugated with cholesterol, increasing siRNA binding to human serum albumin and improving the pharmacokinetic properties when compared to unconjugated siRNA (19). It is important to emphasize that the anti-sense strand, which is responsible for the silencing effect, was not linked to cholesterol, thus making the sense strand a carrier for the antisense.

Clinical trials have already started in humans, targeting the VEGFR1 through intraocular injections of siRNAs to treat agerelated macular degeneration, a deterioration of the retina that is the leading cause of blindness in the elderly (33).

The potential applications of RNAi therapy are extremely broad (34), including all cases in which a deletion or reduction of a defined gene is desired, i.e., genetic diseases, autoimmunity, viral infections, cancer, and many others. The main advances in the use of RNAi have being made in the antiviral and anticancer fields. Although producing efficient antiviral activity, shRNAs targeted to the HIV-2 genome lost their effect due to the emergence of viral variants harboring a point mutation in the shRNA target region. Therefore, as is the case with antiviral drug therapy, RNAi has to be targeted to several genes or 21 mers in the same gene at the same time, preferentially in conserved regions of the viral genome $(35,36)$. However, considering the ease of development of shRNA-expressing plasmids, the specific parts of the viral genome of patients could be sequenced, and the siRNA sequences adapted to the viral genome. This probably is the only technology able to advance faster than both the progression of the disease and the changes in the viral genome, therefore potentially allowing a completely personalized treatment.

Recent developments in the delivery of RNAi to the tumor are a new hope for a highly specific cancer treatment (37), since it theoretically allows the specific targeting of oncogenes present in a given cancer. Targeting oncogenes has already proven to be a very efficient way of fighting cancer since an inhibitor of the $\mathrm{Bcr} / \mathrm{Abl}$ oncogene was able to dramatically increase survival of patients with chronic lymphomas. The development of this drug took years, and such a strategy may not even be applicable to all 
kinds of oncogenes, as indicated by the low efficiency of farnesyltransferase inhibitors in reducing the effects of the ras oncogene. RNAi, on the other hand, can be produced and tested for efficacy within weeks and can be applied to any gene, without exception. An example of RNAi already tested against oncogenes is the siRNA against the RasV12 oncogene, with an A to $\mathrm{T}$ change when compared to its proto-oncogene (17). This siRNA silenced the oncogene without affecting the expression of the proto-oncogene and efficiently blocked the growth of a pancreatic cancer cell line in vivo. As a proof of principle that not only mutations but also translocations can be targeted, a breakpointspecific siRNA was capable of selectively inhibiting Bcr-Abl-dependent cell growth (38).

Methodological advances almost always produce changes in paradigms. The use of RNAi is already producing small changes in paradigms in several fields. For instance, the Akt kinase has been long known to be activated by the phosphorylation of two sites, Thr308 and Ser473. While the kinase that phosphorylates Thr308 has been known for about 10 years, the kinase that phosphorylates Ser473 has remained a mystery despite active research (39). Using mainly RNAi, Sarbassov et al. (40) showed that mTor in complex with the protein Rictor is responsible for the phosphorylation of this site in Akt. mTor was never considered a candidate for the Akt Ser473 kinase because a very specific and efficient inhibitor of mTor, rapamycin, did not reduce the phosphorylation at this site. It turned out that rapamycin only inhibits the mTor/Raptor complex, without affecting the mTor/Rictor complex. RNAi against mTor reduced the phosphorylation of Ser473 of Akt since it eliminated mTor from both complexes, in contrast to rapamycin, which only affected the mTor/Raptor complex.

Science comes in waves of fashionable subjects and sometimes, after the fashion ends, little substance is left. It definitely seems that this is not the case for RNAi and that after the RNAi hype is over we will be left with two very important things: 1) the discovery and description of a fundamental biological mechanism and 2) an easy, fast and reliable way of knocking down a specific gene. If this holds true, RNAi will very soon be part of our everyday lives.

\section{Acknowledgments}

The author wishes to thank Marcia Wink, Fabiana Horn and Pitia Ledur for critical readings of the manuscript and suggestions.

\section{References}

1. Bauocombe D (2004). RNA silencing in plants. Nature, 431: 356363.

2. Fire A, Xu S, Montgomery MK et al. (1998). Potent and specific genetic interference by double-stranded RNA in Caenorhabditis elegans. Nature, 391: 806-811.

3. Timmons L \& Fire A (1998). Specific interference by ingested dsRNA. Nature, 395: 854.

4. Eccleston A \& Eggleston AK (2004). RNA interference. Nature, 431: 337.

5. Mello CC \& Conte Jr D (2004). Revealing the world of RNA interference. Nature, 431: 338-342.

6. Sijen T, Fleenor J, Simmer F et al. (2001). On the role of RNA amplification in dsRNA-triggered gene silencing. Cell, 107: 465-476.

7. Reinhart BJ, Slack FJ, Basson M et al. (2000). The 21-nucleotide let-7 RNA regulates developmental timing in Caenorhabditis elegans. Nature, 403: 901-906.

8. Poy MN, Eliasson L, Krutzfeldt $\mathrm{J}$ et al. (2004). A pancreatic isletspecific microRNA regulates insulin secretion. Nature, 432: 226230.

9. Pfeffer S, Zavolan M, Grasser FA et al. (2004). Identification of virusencoded microRNAs. Science, 304: 734-736.

10. Kennedy S, Wang D \& Ruvkun G (2004). A conserved siRNAdegrading RNase negatively regulates RNA interference in $C$. elegans. Nature, 427: 645-649.

11. Kawasaki H \& Taira K (2004). Induction of DNA methylation and gene silencing by short interfering RNAs in human cells. Nature, 431: 211-217.

12. Stark GR, Kerr IM, Williams BR et al. (1998). How cells respond to 
interferons. Annual Review of Biochemistry, 67: 227-264.

13. Fish RJ \& Kruithof EKO (2004). Short-term cytotoxic effects and long-term instability of RNAi delivered using lentiviral vectors. BMC Molecular Biology, 5: 9-23.

14. Schwarz DS, Hutvagner G, Du T et al. (2003). Asymmetry in the assembly of the RNAi enzyme complex. Cell, 115: 199-208.

15. Khvorova A, Reynolds A \& Jayasena SD (2003). Functional siRNAs and miRNAs exhibit strand bias. Cell, 115: 209-216.

16. Jackson AL, Bartz SR, Schelter J et al. (2003). Expression profiling reveals off-target gene regulation by RNAi. Nature Biotechnology, 21: 635-637.

17. Blummelkamp TR, Bernards R \& Agami R (2002). Stable suppression of tumorigenicity by virus-mediated RNA interference. Cancer Cell, 2: 243-247.

18. Elbashir SM, Harborth J, Lendeckel W et al. (2001). Duplexes of 21nucleotide RNAs mediate RNA interference in cultured mammalian cells. Nature, 411: 494-498.

19. Soutschek J, Akinc A, Bramlage B et al. (2004). Therapeutic silencing of an endogenous gene by systemic administration of modified siRNAs. Nature, 432: 173-178.

20. Myslinski E, Ame JC, Krol A et al. (2001). An unusually compact external promoter for RNA polymerase III transcription of the human H1RNA gene. Nucleic Acids Research, 29: 2502-2509.

21. Tusch $T$ \& Borkhardt A (2002). Small interfering RNAs. Molecular Interventions, 2: 158-167.

22. Hemann MT, Fridman JS, Zilfou JT et al. (2003). An epi-allelic series of p53 hypomorphs created by stable RNAi produces distinct tumor phenotypes in vivo. Nature Genetics, 33: 396-400.

23. Ventura A, Meissner A, Dillon CP et al. (2004). Cre-lox-regulated conditional RNA interference from transgenes. Proceedings of the National Academy of Sciences, USA, 101: 10380-10385.

24. Wiznerowics M \& Trono D (2003). Conditional suppression of cellular genes: lentivirus vector-mediated drug-inducible RNA interference. Journal of Virology, 77: 8957-8961.

25. Rubinson DA, Dillon CP, Kwiatkiwski AV et al. (2003). A lentivirusbased system to functionally silence genes in primary mammalian cells, stem cells and transgenic mice by RNA interference. Nature Genetics, 33: 401-407.

26. Kamath RS, Fraser AG, Dong $Y$ et al. (2003). Systematic functional analysis of the Caenorhabditis elegans genome using RNAi. Nature, 421: 231-236.

27. Ashrafi K, Chang FY, Watts JL et al. (2003). Genome-wide RNAi analysis of Caenorhabditis elegans fat regulatory genes. Nature, 421: 268-272.

28. Kiger AA, Baum B, Jones $S$ et al. (2003). A functional genomic analysis of cell morphology using RNA interference. Journal of Biology, 2: 27-41.

29. Berns K, Hijmans EM, Mullenders J et al. (2004). A large-scale RNAi screen in human cells identifies new components of the p53 pathway. Nature, 428: 431-437.

30. Sen G, Wehrman TS, Myers JW et al. (2004). Restriction enzymegenerated siRNA (REGS) vector and libraries. Nature Genetics, 36 : 183-189.

31. Shirane D, Sugao K, Namiki S et al. (2004). Enzymatic production of
RNAi libraries from cDNAs. Nature Genetics, 36: 190-196.

32. Luo B, Heard AD \& Lodish HF (2004). Small interfering RNA production by enzymatic engineering of DNA (SPEED). Proceedings of the National Academy of Sciences, USA, 101: 5494-5499.

33. Check E (2004). Hopes rise from RNA therapy as mouse study hits target. Nature, 432: 136.

34. Shankar P, Manjunath N \& Lieberman J (2005). The prospect of silencing disease using RNA interference. Journal of the American Medical Association, 293: 1367-1373.

35. Gitlin L, Karelsky S \& Andino R (2002). Short-interfering RNA confers intracellular antiviral immunity in human cells. Nature, 418: 430434.

36. Boden D, Pusch O \& Lee F (2003). Human immunodeficiency virus type 1 escape from RNA interference. Journal of Virology, 77: 1153111535.

37. Tong AW, Zhang YA \& Nemunaitis J (2005). Small interfering RNA for experimental cancer therapy. Current Opinion in Molecular Therapeutics, 7: 114-124.

38. Wohlbold L, Van der Kuip H, Vornlocker HP et al. (2003). Inhibition of bcr-abl gene expression by small interfering RNA sensitizes for imatinib mesylate (STI571). Blood, 102: 2236-2239.

39. Bayascas JR \& Alessi DR (2005). DR regulation of Akt/PKB Ser473 phosphorylation. Molecular Cell, 18: 143-145.

40. Sarbassov DD, Guertin DA, Ali SM et al. (2005). Phosphorylation and regulation of Akt/PKB by the rictor-mTOR complex. Science, 307: 1098-1101.

41. Lau NC, Lim LP, Weinstein EG et al. (2001). An abundant class of tiny RNAs with probable regulatory roles in Caenorhabditis elegans. Science, 294: 858-862.

42. Lee RC \& Ambros V (2001). An extensive class of small RNAs in Caenorhabditis elegans. Science, 294: 862-864

43. Paddison PJ, Caudy AA \& Hannon G (2002). Stable suppression of gene expression by RNAi in mammalian cells. Proceedings of the National Academy of Sciences, USA, 99: 1443-1448.

44. Blummelkamp TR, Bernards R \& Agami R (2002). A system for stable expression of short interfering RNAs in mammalian cells. Science, 296: 550-553.

45. Paddison PJ, Caudy AA, Bernstein E et al. (2002). Short hairpin RNAs (shRNAs) induce sequence-specific silencing in mammalian cells. Genes and Development, 16: 948-958.

46. Miyagishi M \& Taira K (2002). U6 promoter-driven siRNA with four uridine 3' overhangs efficiently suppress targeted gene suppression in mammalian cells. Nature Biotechnology, 20: 497-500.

47. Lee NS, Dohjima T, Bauer G et al. (2002). Expression of small interfering RNAs targeted against HIV-1 rev transcripts in human cells. Nature Biotechnology, 20: 500-505.

48. Paul CP, Good PD, Winer I et al. (2002). Effective expression of small interfering RNA in human cells. Nature Biotechnology, 20: 505-508.

49. Xia $\mathrm{H}$, Mao $\mathrm{Q}$, Paulson $\mathrm{HL}$ et al. (2002). siRNA-mediated gene silencing in vitro and in vivo. Nature Biotechnology, 20: 1006-1010.

50. Stewart SA, Dykxhoorn DM, Palliser D et al. (2003). Lentivirusdelivered stable gene silencing by RNAi in primary cells. RNA, 9: 493-501. 